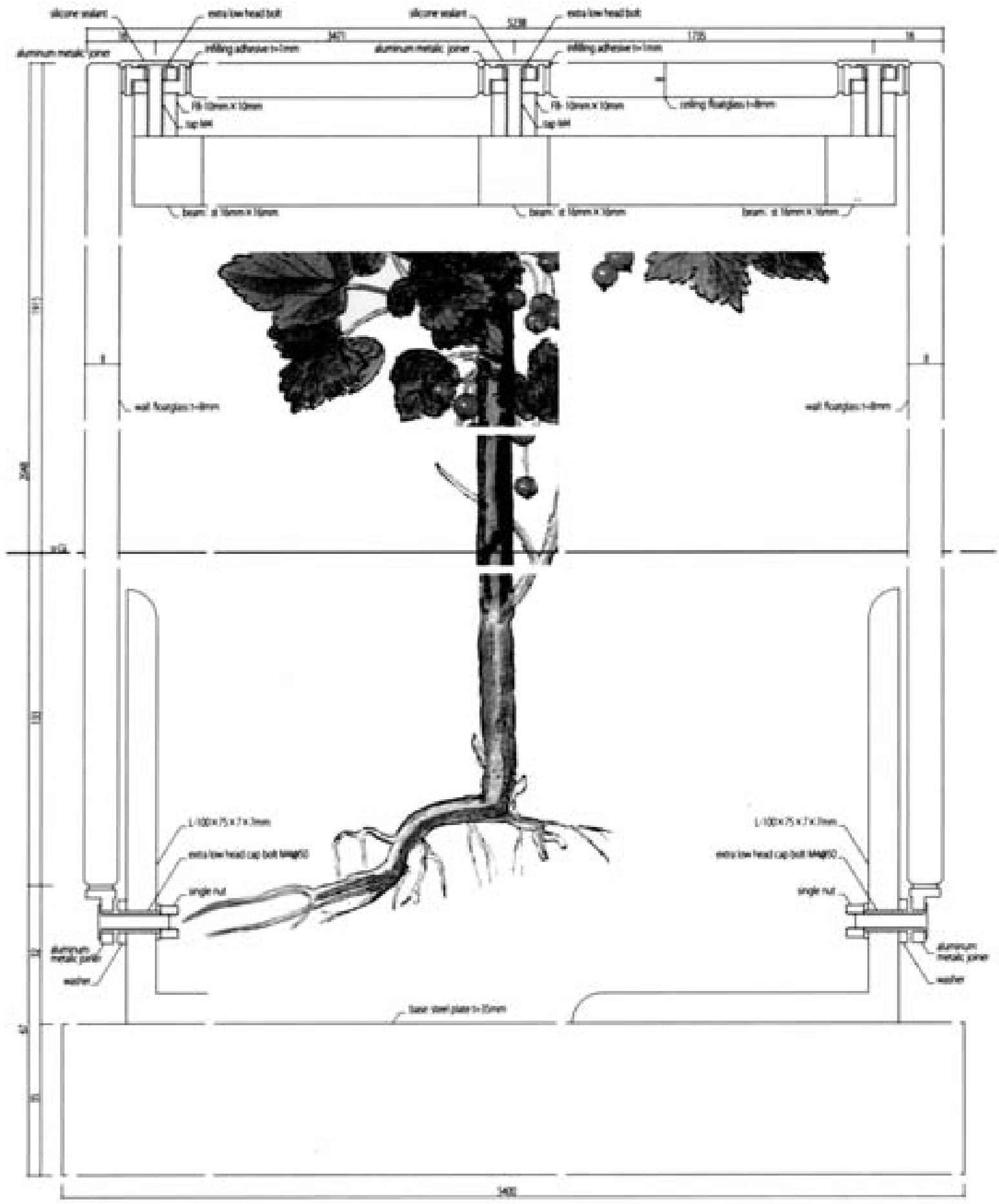




\section{La naturaleza como}

\section{experimento constructivo. El Pabellón de Japón para la XI Bienal de Venecia (Junya Ishigami, 2008) Ángela Juarranz}

El trabajo de Junya Ishigami ha sido objeto de estudio frecuente en el marco de una arquitectura próxima a la naturaleza, interés compartido por la constelación de arquitectos japoneses de finales del siglo $X X$ principios del XXI. Este artículo estudia la obra de Ishigami de manera más específica en torno a la condición natural como laboratorio de construcción, acorde a una doble finalidad con piezas destinadas a exponerse en galerías o a formalizarse como arquitectura. Esta dedicación paralela permite discutir la operatividad de unas estrategias enfocadas a la ejecución material de un medio permanente que, sin embargo, sólo alcanzan su objetivo en forma de instalación de arte temporal. Como caso de estudio se considera Extreme Nature: Landscape of Ambiguous Spaces, el proyecto diseñado por Ishigami para el Pabellón de Japón en la 11a Mostra Internazionale di Architettura de la Biennale di Venezia de 2008.

\section{PALABRAS CLAVE}

Naturaleza, material, Junya Ishigami, Pabellón de Japón, Bienal de Venecia

\section{KEYWORDS}

Nature, Material, Junya Ishigami, Japanese Pavilion, Venice Biennial

ENTRE

ARQUITECTURA

Y PAISAJE, LA

DIFUSIÓN DEL

LÍMITE

\section{Extreme Nature: Landscape of Ambiguous}

Spaces fue la instalación diseñada por el arquitecto Junya Ishigami (Kanazawa, 1974) para el Pabellón de Japón en la 11ª Mostra Internazionale di Architettura de la Biennale di Venezia de 2008. La propuesta de Ishigami, el más joven de la "constelación japonesa", además de representar el tema "Out There: Architecture Beyond Building" enunciado por el director de la bienal Aaron Betsky, proponía espacios que vaticinaban una nueva arquitectura. Cuatro estructuras de metal y vidrio ubicadas en el jardín del pabellón asumían la función de invernaderos al mismo tiempo que constituían un paisaje complemento del anterior. Las piezas contenían diferentes
Ángela Juarranz Serrano

Arquitecto (2012) y Máster en Proyectos Arquitectónicos (2013) por la ETS de la Universidad Politécnica de Madrid. Es Profesor en Formación en el Departamento de Proyectos de la ETSAM, donde realiza la tesis doctoral bajo el Convenio FPU del Ministerio de Educación, Cultura y Deporte. Ha sido Visiting Scholar en la Universidad de Columbia en Nueva York gracias a la Beca de Investigación de la Fundación Arquia y la Real Academia de Bellas Artes de San Fernando. Su actividad investigadora ha sido publicada por Cuadernos de Proyectos Arquitectónicos (2018), [i2] Innovación e Investigación en Arquitectura y Territorio (2017), International Institute for Conservation of Historic and Artistic Works (2016), la Biena Internacional de Arquitectura de Argentina (2014) y el Círculo de Bellas Artes de Madrid (2013). Su obra ha sido reconocida por la Bienal Española de Arquitectura y Urbanismo (2018), el Colegio Oficial de Arquitectos de Madrid (Premio COAM 2017 y Premio PFC COAM 2013), el Colegio Oficial de Arquitectos de España (Europan 12 y 13) y las publicaciones Bauwelt (First Works Awards 2017) y Arquitectura Viva, entre otros.

Orcid ID 0000-0002-3520-4754

Fig. 01

ISHIGAMI, J., Detalle constructivo del 'Pabellón de Japón', 2008. 
Fig. 02

ISHIGAMI, J., ‘Pabellón de Japón', 2008.

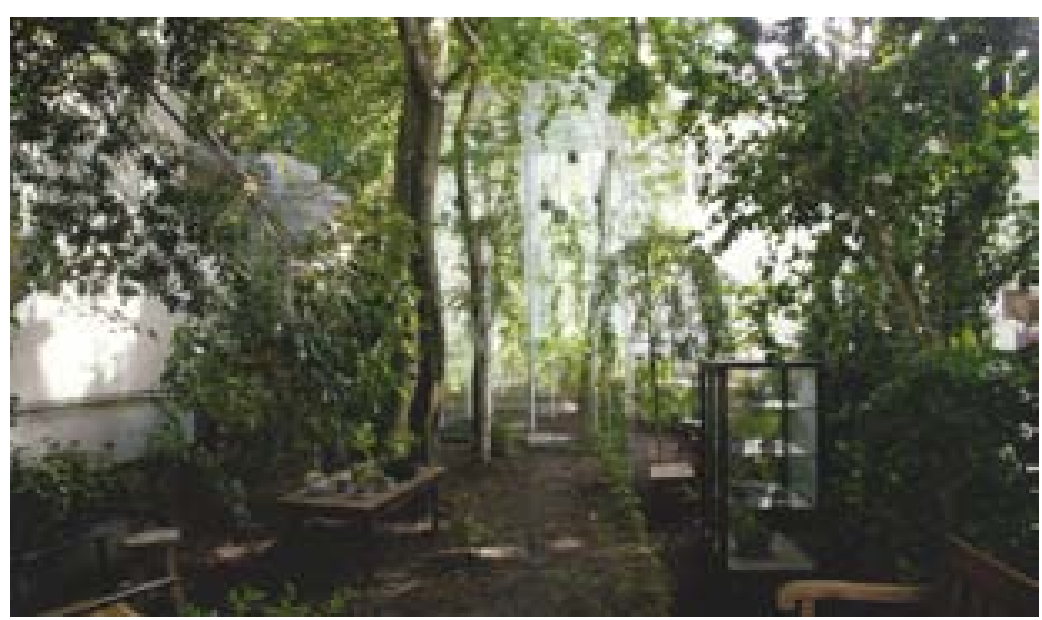

02

ecosistemas de modo que sus medidas dependían del crecimiento y de las necesidades lumínicas de las plantas. Además, la concentración de vegetación de cada estancia era tal que la densidad creada en el interior y el exterior resultaba equivalente. Las fotografías tomadas en el jardín muestran cómo los invernaderos, a causa de las dimensiones, la materialidad y la atmósfera, difuminaban los límites de los volúmenes y definían un paisaje nuevo y unificado (fig. 02).

La parte de la intervención ubicada dentro del pabellón seguía la idea de proximidad con la naturaleza. Dentro de un espacio completamente vacío, a excepción de unas sillas blancas, las paredes mostraban unos dibujos que relacionaban la ciudad y el paisaje. Las imágenes consistían en una secuencia de visiones utópicas de la metrópoli del futuro, acompañadas de unas inscripciones que describían las nuevas tipologías edificatorias. Estas pinturas presentaban tipos como los Plant Buildings, unas parcelas de tierra donde la naturaleza salvaje crecía libremente dentro del área urbana, o los Greenhouse Buildings, unos bloques formados por el apilamiento de ecosistemas diferentes según el medio y la altura en que se situaban². En esos dibujos, el ajetreo de la ciudad, la intimidad del hogar y la sensibilidad del parque confluían en una única entidad. La intervención, tanto en el interior como exterior del pabellón, imaginaba una arquitectura donde la construcción, el mobiliario y el paisaje transcendían las categorías relativas y se entremezclaban libremente. El difuso límite entre las piezas y el paisaje abría un camino en el que investigar nuevas relaciones espaciales y materiales, con un diseño y técnica propios.

La condición experimental del Pabellón de Venecia hace pertinente estudiar la obra de Ishigami desde el proceso de ideación y en base a una disciplina doble, a medio camino entre el arte y la arquitectura ${ }^{3}$. Por ejemplo, el grado de minuciosidad que requirió la instalación de Venecia, desde el desarrollo de la idea hasta la ejecución, responde más al carácter obsesivo de la producción artística que al rigor técnico del dibujo de arquitectura. El proyecto se basó en los planos del arquitecto Takamasa Yoshizaka para la construcción del Pabellón de Japón en 1956. Las fotos que documentan el proceso de diseño muestran unos modelos a escala de los invernaderos que se distribuyen sobre la reimpresión del dibujo de Yoshizaka, respetando pero tensando la relación entre los volúmenes y la naturaleza, jugando con la distribución de los 
invernaderos, la vegetación, los claros, las rocas y los caminos (fig. 03). Yoshizaka diseñó el pabellón con tal coherencia que es difícil detectar una clara diferencia entre interior y exterior; edificio y paisaje se entienden como una única pieza. Con ese fin, el pabellón se apoya puntualmente sobre columnas, propicia los recorridos múltiples en la planta inferior y posibilita la relación del volumen con la colina de los jardines. Tomar como punto de partida el trabajo de Yoshizaka hizo que todos esos parámetros de experiencia se incorporasen en el interior y el exterior en la nueva propuesta (figs. 04 y 05 ).

Las instantáneas tomadas por los visitantes del pabellón plasman la condición diáfana de toda la instalación y la posición coordinada entre todos los elementos. En el jardín, en torno a los invernaderos y los árboles preexistentes, se dispusieron varias piezas de mobiliario y numerosas macetas cerámicas. Los dibujos del proyecto junto con las innumerables instrucciones dadas durante el montaje de la exposición son signo de la importancia que tenía cada elemento ${ }^{4}$. Definir con minuciosidad todos los objetos del espacio permitía que cada pieza entrase en valor al unísono: las plantas, las sillas, la vitrina, las cerámicas, las piedras, el terreno y la vegetación original. La ejecución del nuevo jardín, acorde a los objetivos de la maqueta de papel, logró generar en la instalación unos nuevos registros arquitectónicos.

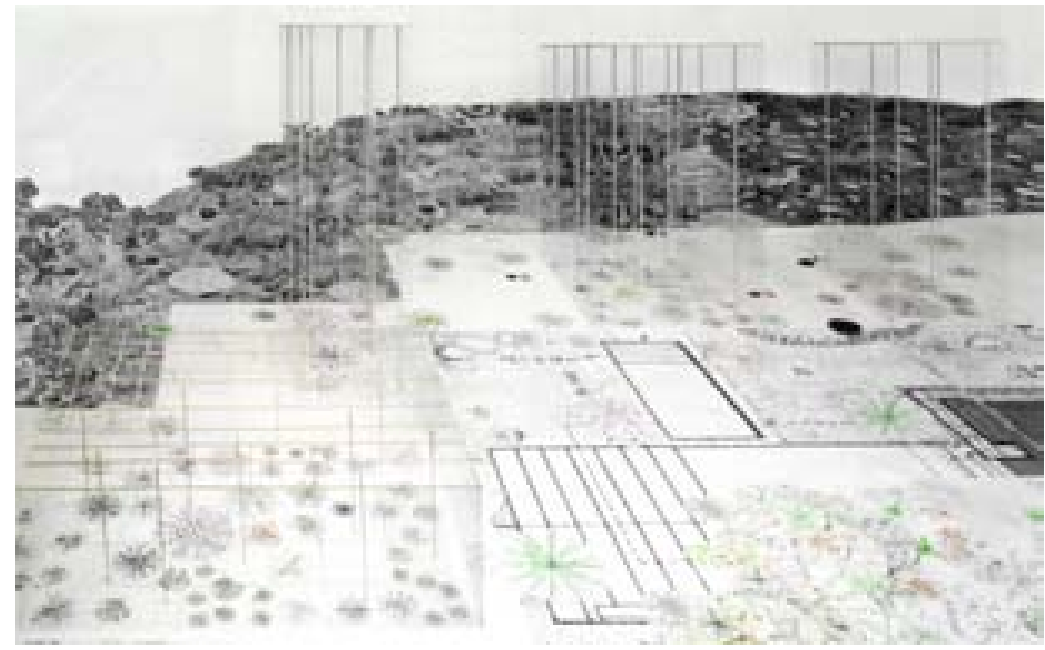

03

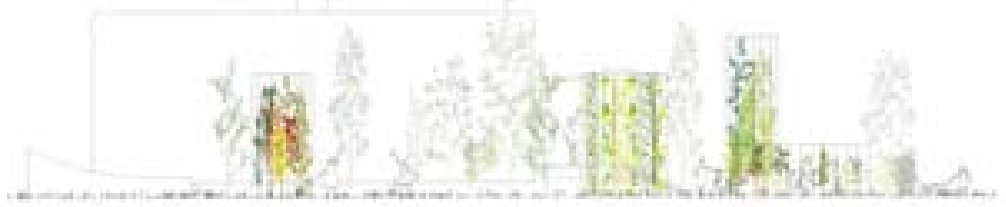

04

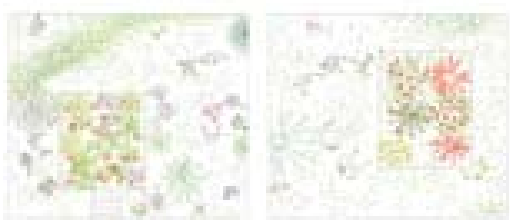

Figs. 03, 04 y 05

ISHIGAMI, J., Maqueta, alzado y planta del 'Pabellón de Japón', 2008. 
LA CONDICIÓN

PLURIDISCIPLINAR:

DE LA BOTÁNICA A LA

ARQUITECTURA
Las fotos, dibujos y textos que documentan la construcción del Pabellón reflejan un conocimiento pluridisciplinar. A través del dominio de la botánica, la solución de los invernaderos no se dirigía a crear un hábitat extremo como los jardines que albergan plantas tropicales en climas fríos. Ni siquiera estaban dotados de reguladores térmicos u otros sistemas de acondicionamiento de aire. Se diseñaron en el contexto de una arquitectura experimental que buscaba unos cambios de temperatura y humedad mínimos. Para algunas plantas, esta variación de temperatura suponía una desviación tan leve que podían crecer tanto en el interior como en el exterior de la estructura, generando un único ambiente y diluyendo la condición funcional del invernadero. Según las pautas del botánico colaborador Hideaki Oba ${ }^{5}$, se seleccionaron plantas que tal vez crecieron de forma natural en Venecia, o potencialmente podrían hacerlo con los cambios climáticos propios de la ciudad'. La delicadeza con la que se cultivaron recuerda las labores tradicionales de la cultura japonesa, como la ikebana, el arte japonés de arreglo floral, y el shakkei, la creación de pequeños paisajes realistas ${ }^{7}$. Esta actitud resulta próxima a la teoría de John Dixon Hunt que, frente a la jardinería como actividad práctica, enfatizaba la base teórica y conceptual del arte en el jardín. Según Hunt, esto implicaba entender el jardín en el contexto cultural a lo largo de diferentes épocas y lugares y tratarlo como el epítome de la creación de un nuevo entorno. Ciertamente, la instalación, tanto en el diseño del paisaje como en el desarrollo técnico de las piezas, perseguía unos nuevos contextos habitables. La instalación de Ishigami, aún bajo la condición de ser un elemento temporal y sin una función definida, inauguraba unas líneas de investigación comprometidas con la búsqueda de nuevas arquitecturas.

La definición técnica del Pabellón incide en los intereses explorados durante el proceso de diseño, como la conexión física y la similitud de temperatura entre interior y exterior. La radicalidad de los objetivos de partida requirió de unos conocimientos estructurales específicos. Los volúmenes se realizaron con un entramado estructural metálico blanco formado por pilares y vigas de sección cuadrada de 16 milímetros de lado y una piel de vidrio flotado de 8 milímetros de grosor (fig. 06). Los pilares, tanto en posición como en dimensión, venían determinados por un análisis estructural que minimizaba las cargas y esfuerzos de la pieza, al igual que las vigas, con un intereje que variaba entre 20 y 85 centímetros. A su vez, los vidrios perimetrales colgaban de la parrilla de vigas y cumplían el papel de tirantes que absorbían las cargas horizontales del pabellón. La unión entre la estructura de acero y el vidrio se fijaba mediante unas piezas metálicas mínimas que aseguraban mediante tornillería la estabilidad de un volumen de 20 metros cuadrados de planta y 2 metros de altura que, compactado, apenas sumaba 0,3 metros cúbicos y 800 kilos de peso propio. Con el fin de ocultar la cimentación y los sistemas de riego instalados sobre rasante, se añadió y embelleció la superficie con una nueva capa de tierra que superaba el medio metro y que tuvo que extenderse por el total del área japonés para ajustar la topografía del nuevo paisaje. Cada uno de estos elementos fue diseñado para participar mecánicamente, pero a su vez adquirían la escala propicia para formar parte del tejido natural. En vez de crear un entorno extraño, los invernaderos y las plantas se entremezclaban en el medio existente. La construcción confirmaba el interés de Ishigami por reunir el lenguaje estructural de la arquitectura y la imagen y percepción de la naturaleza. En lugar de la implicación formal o, incluso, ecológica 


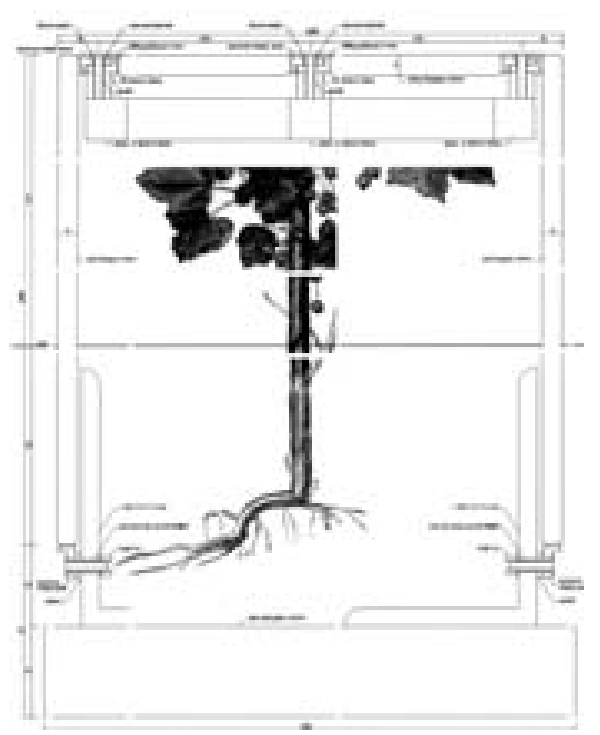

06

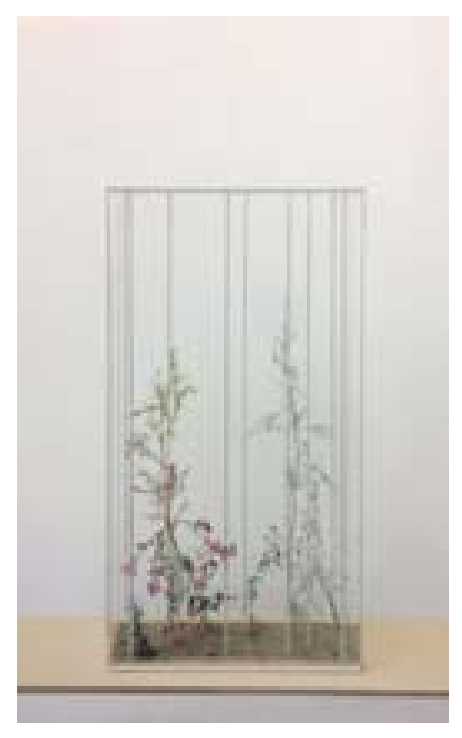

07

de la geometría orgánica, el arquitecto meditaba en torno a la poética de las estructuras naturales y físicas. Podría decirse, que en este caso la investigación del autor era más sensorial que pragmática.

En la labor dual de Ishigami como artista y como arquitecto, su producción manifiesta unas cualidades arquitectónicas, pero también revela la preeminencia de un valor escultórico. Dentro del contexto de una arquitectura como parte del paisaje, la realización de prototipos y la estrategia proyectual invitan a profundizar en la doble condición del autor. Por un lado, el desarrollo de prototipos se caracteriza por una ejecución minuciosa y artesana mediante maquetas que reproducen de manera obsesiva temas relacionados con la naturaleza ${ }^{10}$. A menudo, el destino de estos modelos ha sido su exposición en galerías y museos de arte. Tanto los pequeños objetos mostrados en las salas de exposición como las arquitecturas construidas confirman un proceso de experimentación común. Por ejemplo, la investigación realizada para el Pabellón de Japón se completó con las maquetas Greenhouses, de estructura ligera y de diferentes formas y proporciones, que exhibieron la nueva estrategia arquitectónica en el interior de las galerías Toyota Municipal Museum of Art (Toyota, 2010), Shiseido Gallery (Tokio, 2010), deSingel (Amberes, 2013) y Arc en Rêve (Burdeos, 2014)" (fig. 07). Como contrapunto, la extrema esbeltez de los modelos $S k y$ (2010) redujo la viabilidad de esa propuesta a una reproducción de escala 1/3000. Ahí, la limitación de la tecnología contemporánea impedía la traslación directa de una noción natural más próxima a lo escultórico que a la solución realista'2.

En cuanto a la estrategia proyectual, el grado de libertad con que Ishigami inicia los encargos marca un protocolo metodológico que inaugura nuevas líneas de investigación. En cada proyecto se acerca a valores irregulares, inestables e inciertos con el objetivo de liberar la arquitectura de conceptos preconcebidos, como tipologías uniformes o estilos concretos. Las nuevas propuestas buscan una alternativa al incorporar parámetros pasados o futuros, regionales o foráneos, arquitectónicos o ajenos $^{13}$. Para ello, Ishigami justifica una flexibilidad entendida como autonomía; que opera a modo de búsqueda genuina e incorpora tanto aspectos

\section{Fig. 06}

ISHIGAMI, J., Detalle constructivo del 'Pabellón de Japón', 2008.

Fig. 07

ISHIGAMI, J., ‘Greenhouses', 2010. 


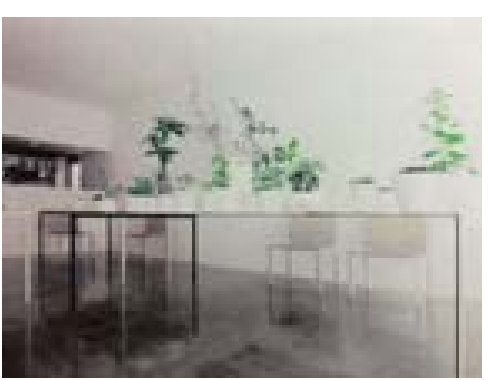

08

Fig. 08

ISHIGAMI, J., 'Tables for a Restaurant', 2008. específicos como abstractos. El proyecto del Pabellón de Venecia surge de esos grados de libertad que quieren alejarse de una arquitectura preconcebida y enfatizar la materialidad natural del pabellón. En primer lugar, la disolución del invernadero en la naturaleza introduce el tema de la libertad de entorno. La extrapolación del escenario doméstico, urbano y natural por medio de un espacio compartido manifiesta la libertad de escala. $Y$ por último, la emancipación de usos y la apropiación libre del paisaje por parte del usuario significan la libertad de función. En la instalación de Venecia, estas estrategias sí son transcritas a la escala real pero en otros casos permanecen como idealizaciones de otra arquitectura posible.
LA CONDICIÓN NATURAL PARA UNA LIBERTAD DE ENTORNO, DE ESCALA Y DE FUNCIÓN
El estudio de la operatividad de las propuestas artísticas y arquitectónicas de Ishigami descubre una lectura crítica sobre las condiciones de entorno, de escala y de función de la cualidad natural. En cuanto al entorno, la obra de Ishigami incorpora nociones que hacen referencia a campos, bosques, montañas, valles, ríos, lagos, nubes y niebla. A modo de experimentos, estos sistemas existen como temas que más tarde se transfieren a la arquitectura. Lo singular de las piezas resultantes es el hecho de que los fenómenos naturales son el material con el que se construye la arquitectura. Al contrario que el supuesto común donde la arquitectura es el refugio que nos aísla del contexto, ahora lo natural es parte del medio diseñado. Esa inversión posibilita un espacio donde artificio y naturaleza confluyen. En el Pabellón de Venecia el entorno que se establece en virtud de la influencia mutua entre lo artificial y natural genera el espacio como tal, libre de distinguir entre una condición u otra. Caminar por el jardín o entrar y salir de los invernaderos no respondía al sentido de estar dentro o fuera. Siguiendo su protocolo exploratorio, la instalación modificaba el fin de refugio para generar junto con el paisaje circundante un nuevo medio.

Al igual que el Pabellón de Venecia, otros proyectos de Ishigami revisan los modos de relación entre arquitectura y naturaleza. Tables for a Restaurant (2005) y House with Plants (2012) plantean sistemas de organización espacial donde las plantas caracterizan ámbitos de espacio exclusivo sobre la trama artificial. Tables for a Restaurant consiste en el diseño y disposición de unas mesas para varias parejas de comensales. Cada tablero tiene una superficie muy amplia colmatada de numerosas macetas que aúna la condición natural y los requisitos programáticos (fig. 08). En la casa House with Plants la tierra entra en el interior del edificio y crea una sucesión de espacios verdes habitables. La frontera entre el contexto doméstico y el paisaje se diluye y origina un ámbito de carácter doble. En esta casa, la libertad de entorno, o en otras palabras, la incorporación de la naturaleza, es efectiva tanto en los modelos a escala como en la arquitectura final. A su vez, esta incorporación de lo natural puede entenderse no como el logro de la experimentación con procesos vinculados al arte, sino como un eco de los denominadores comunes de la "constelación japonesa", con ejemplos como Teshima Art Museum (2010) o Garden and House (2013) de Ryue Nishizawa.

En cuanto a la escala, Ishigami la entiende como el alcance que llega a tener la percepción humana, tanto en la apreciación a gran distancia como en la de las cosas más pequeñas. Ante la grandiosidad de la máquina como protagonista de épocas pasadas, antepone un contexto 
natural que contiene a la vez, y a partes iguales, lo indómito y lo delicado. Las escalas de los espacios de la naturaleza se extienden sin límites entre ambos puntos: la infinitud del cielo, la ligereza de una nube, la nimiedad de una gota de agua. En el Pabellón de Venecia, la estructura ligera y la densidad de plantas son pautas que enfatizan esa libertad de escala.

Proyectos como Little Gardens (2007) y Island Garden (2010) también enfatizan la amplitud de escalas en el entorno natural'14. En Little gardens, 370 vasijas de apenas unos centímetros de tamaño funcionan como cuencos donde exhibir flores prensadas. La imagen total parece la de una ciudad formada por sucesivos terrenos ajardinados (fig. 09). La propuesta /slands Gardens, a modo de paradigma urbano de Little Garden, consiste en un grupo de islas que ocupan un lago por medio de un sistema de parcelación. La maqueta refleja un paisaje compuesto por láminas flotantes disponibles para las futuras construcciones. Estos estudios enfatizan el vínculo entre el escenario y la ocupación a nivel cuantitativo, pero obvian otras condiciones intrínsecas a la escala. Reducir el concepto de escala a la variable dimensional implica perder otros aspectos como el vital, el ecosistémico, el virtual o el de información, que son, en cambio, más acordes a la conciencia medioambiental contemporánea. El acercamiento metodológico de Ishigami a través de la libertad de escala remite a una maniobra de abstracción con la que las intenciones estructurantes se priorizan sobre la ecología.

Ishigami introduce una variable más. A través de la caracterización del espacio entre binomios como grande y frágil, extenso y bajo o alto y estrecho, incorpora contenidos de formas diversas y programas complejos; hay confort y tensión, claridad y vaguedad, amplitud y estrechez. Ante las situaciones de cambio en la actividad económica, política o personal, Ishigami propone unos espacios basados en la adaptabilidad con una tolerancia capaz de acomodar casi cualquier uso. Reclama un sistema flexible como instrumento idóneo para que la arquitectura lidie con la velocidad y aleatoriedad del mundo actual. No considera lo flexible en el marco

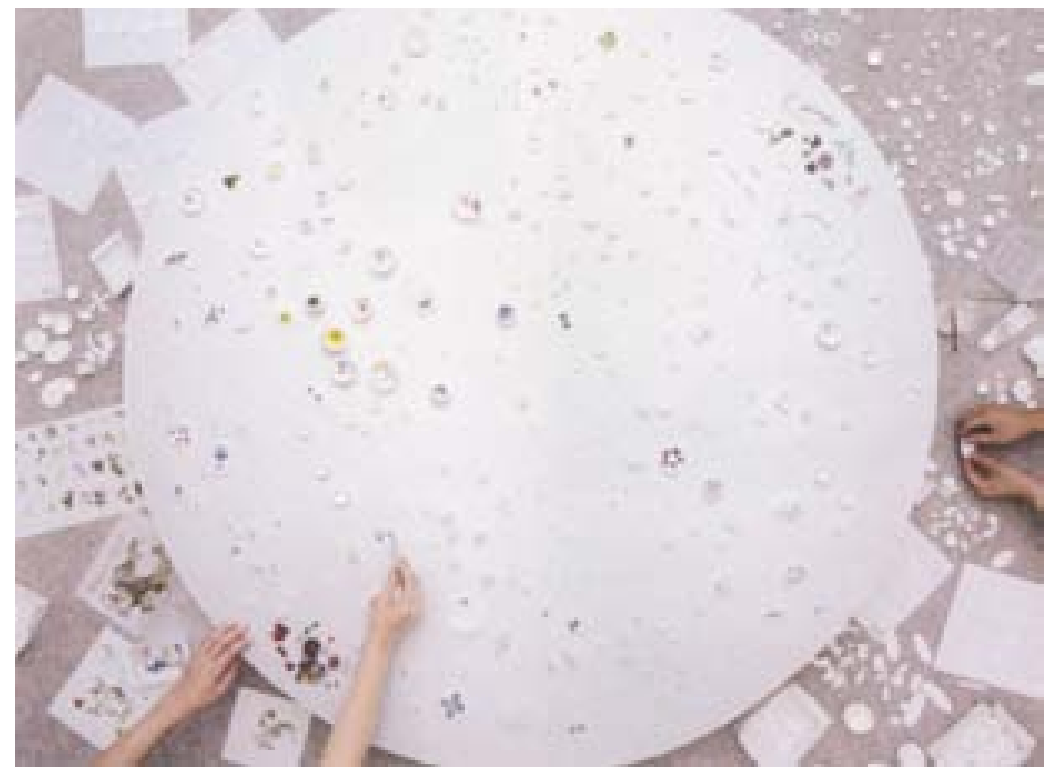




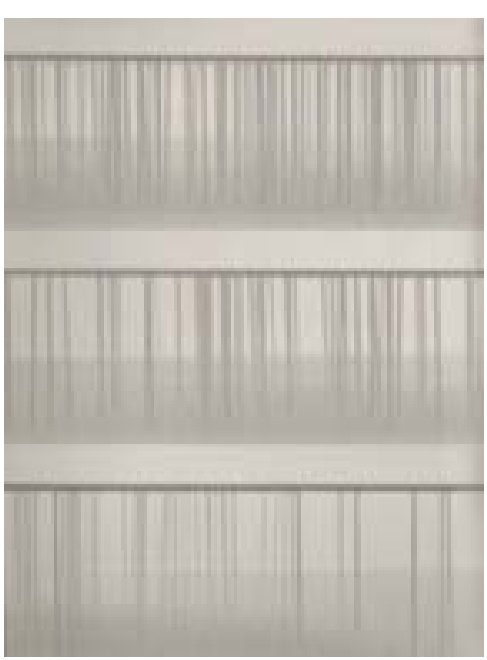

10 de las expectativas, sino en la "no-uniformidad"15 propia de fenómenos naturales, y en una "incertidumbre"16 en la que el propio sistema de cambio se halla en estado de transición. Con esta medida evita la aplicación de funciones uniformes y arquitecturas estándares. Sin un objetivo determinado, el Pabellón de Venecia consiste en una apropiación del paisaje, de función abierta, a medio camino entre lo urbano, lo doméstico y lo íntimo.

Ejemplos como Forest (2008) y KAIT Workshop (2008) conciben el espacio como un bosque, un lugar multiusos del que apropiarse libremente. La maqueta Forest visibiliza cómo el crecimiento de un bosque está condicionado por la agrupación o dispersión de la vegetación (fig. 10). Por ejemplo, dependiendo de la densidad de plantas en un parque, se reproducen unas u otras variedades, con un porte mayor o menor. En el edificio $K A I T$, el espacio para talleres anejo a la Universidad de Kanazawa, la ambigüedad del bosque se traduce en un principio orgánico que distribuye el espacio. El valor del edificio reside en la condición fluctuante de función, dependiente de la forma y de la dimensión de los espacios, y de cómo éstos están unidos y separados. En este caso, tanto la instalación de arte Forest como la obra construida $K A / T$, materializan la libertad de función del medio natural y generan una nueva estrategia de ocupación. Esa concepción de libertad de función no sólo caracteriza la obra de Ishigami, sino que se relaciona con el concepto de lo "público" en el contexto de la arquitectura japonesa contemporánea. En japonés, el término "público" está relacionado con el uso y la ocupación del espacio, que incorpora nociones como la apertura física, la espontaneidad funcional y los procesos de decisión popular ${ }^{17}$, características clave del Rolex Learning Center (2010) de SANAA.

LA NATURALEZA
COMO
LABORATORIO
DE UNA
CONSTRUCCIÓN
TEMPORAL

En el logro de la libertad de entorno, de escala y de función, y gracias a unos procesos de trabajo pluridisciplinares, Ishigami investiga una arquitectura de carácter intrínsecamente natural. Frente a una arquitectura sometida a regulaciones normativas, económicas y sociales, introduce un protocolo que reformula esas preocupaciones y explora una materialización diferente. Tal es el caso del Pabellón de Japón y los grados de autonomía con los que se construye el proyecto. En su diseño, a modo de arquetipo, Ishigami despliega las posibilidades de la naturaleza como nuevo material de construcción ${ }^{18}$ (fig. 11).

Cabría una última reflexión a propósito de los grados de libertad de la instalación de arte y de su operatividad como gestores de una nueva arquitectura natural. En la oficina de Ishigami, las maquetas, más allá de ser meras herramientas de trabajo, son piezas con una investigación y una formalización final. Por ejemplo, en las maquetas Greenhouses se explora la esbeltez y transparencia de los invernaderos y se logra reinterpretar la relación entre personas, pabellón y entorno. En cambio, los modelos diseñados para oscilar frente al viento, que constituyen una tipología de comportamiento estructural similar al de las especies naturales, resultan de dudosa aplicación directa en arquitectura. También, como un juego óptico, se realizan fotomontajes imposibles a partir de fotografías de maquetas tomadas desde diferentes puntos de vista. El hecho de que estas piezas se produzcan por medio del distanciamiento sobre requisitos de entorno, escala y función, valores intrínsecos a la arquitectura, parece dificultar la transcripción de algunos de los conceptos de la naturaleza en obras concretas. En la búsqueda hacia una nueva arquitectura, Ishigami enuncia: "Me gustaría explorar la
Fig. 11
ISHIGAMI, J., 'Pabellón de Japón', 2008.

Fig. 10

ISHIGAMI, J., ‘Forest', 2010. 
posibilidad de transformar en arquitectura aquello que nunca antes ha sido arquitectura. Probablemente, esto signifique repensar los métodos con los que construir arquitectura. Al hacer esto, seguramente descubriremos un

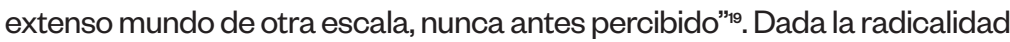
de esos intereses, gran parte de los planteamientos se exhiben en las exposiciones temporales, pero fallan en su consolidación como diseños de arquitectura permanentes. Esto justifica el éxito de instalaciones como Magic Table (2005), Cuboid Balloon (2008) o Extreme Nature (2008) pero remarca la limitación técnica de los proyectos Greenhouse Building (2008), Plant Building (2008) y Island Gardens (2010). Dos años después de la exhibición Extreme Nature, el equipo de Ishigami volvía a la Bienal de Venecia con la obra Architecture as Air: Study for Château La Coste. La instalación, inspirada en una condición etérea natural y ejecutada con unos filamentos de fibra de carbono finos y esbeltos, apenas visibles, colapsaba unas horas antes de la inauguración. Días más tarde, Ishigami era galardonado con el León de Oro al mejor proyecto de la muestra por superar "los límites de la materialidad, la visibilidad, la tectónica, la ligereza y la propia arquitectura". El proyecto era un ensayo a escala real para un futuro pabellón del complejo Château La Coste. El éxito como instalación temporal pone de manifiesto una experimentación que busca el avance de la disciplina a través de la lógica natural, pero que fracasa en el intento de asegurar la supervivencia bajo la condición de permanencia que la arquitectura impone. KAIT Workshop (2008) o House with Plants (2012) sí se consolidan como arquitecturas referentes del siglo XXI, aunque, por ahora, el alcance de estas obras parece más la continuación de los anhelos de la "constelación japonesa", y no tanto el logro constructivo de los imaginarios naturales más radicales. RA

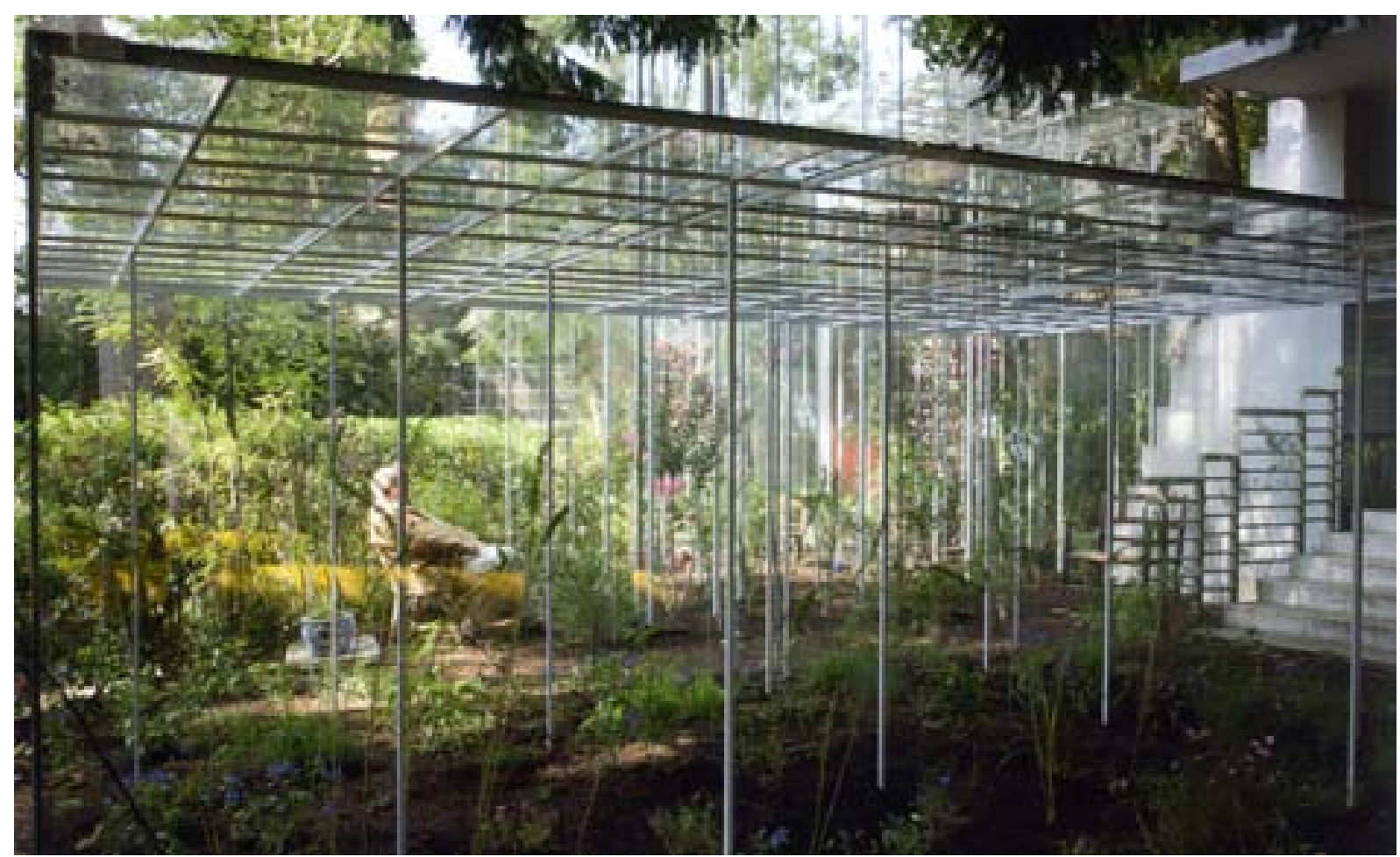


01. La exposición “A Japanese Constellation" (2015), realizada en el The Museum of Modern Art y comisariada por Pedro Gadanho, incluyó la obra de los arquitectos Toyo Ito, Kazuyo Sejima, SANAA, Ryue Nishizawa, Sou Fujimoto, Akihisa Hirata y Junya Ishigami. En el artículo "The Deep Field: Resolving a Japanese Constellation", incluido en el catálogo de la exposición, el arquitecto y crítico Julian Worrall, especializado en arquitectura japonesa contemporánea, caracteriza la arquitectura de esta generación bajo unos intereses comunes por lo natural, lo público, la ligereza y la abstracción. GADANHO, Pedro, $A$ Japanese Constellation, New York City, The Museum of Modern Art, 2015, pp. 245-249.

02. ISHIGAMI, Junya, "Greenhouse designing with Yoshizaka", En JA 79, Junya Ishigami, 2010, 79, p. 40, 43.

03. Taro Igarashi indica el interés de Ishigami por el arte visual no sólo a causa de la dificultad de los arquitectos japoneses por encontrar trabajo tras la burbuja económica de los años 1980, sino también por su entendimiento de la arquitectura como utopía. Ishigami imagina las instalaciones temporales como otro tipo de arquitectura. Progresivamente, algunas de las investigaciones sí han podido adaptarse a diseños concretos y permanentes. IGARASHI, Taro, "A Few Things I Know About Junya Ishigami”. En Junya, Junya Ishigami: another scale of architecture. Kyoto, Seigensha Art Publishing, 2010. pp. 270-289.

04. IGARASHI, Taro, "A Few Things I Know About Junya Ishigami". En Junya. Junya Ishigami: another scale of architecture. Kyoto, Seigensha Art Publishing, 2010, p. 284.

05. Hideaki Oba es Profesor en el Departamento de Botánica de la Universidad de Tokyo y ex-director del Jardín Botánico Koishikawa. Fue también colaborador de Junya Ishigami en la instalación Balloon (2007) para el Museum of Contemporary Art de Tokio.

06. Los planos de ejecución acotaban la posición y especie de cada invernadero. Entre otras, el más esbelto de los cuatro recintos $(1,9 m \times 3,2 m \times 6,1 \mathrm{~m})$ incluía plantas de porte esbelto y flores de climas húmedos como gledtsia triacanthos, bougainvillea, cyperus papyrus y acacia saligna. El más bajo $(5,2 \mathrm{~m} \times 3,8 \mathrm{~m} \times 2,1 \mathrm{~m})$ contenía flora nativa de las regiones tropicales de Sudamérica y América Central como solanum jasminoides, cleome spinosa, echium fastuosum y púnica granatum. Los de proporción media (3,1m $\times 3,5 m \times 4,7 m$ y $2,2 m \times 2,7 m \times 4,7 m$ ) tenían plantas húmedas nativas del hemisferio norte como jasminium officinalis, rosa banksiae, hederea hélix y mandevilla boliviensis. ISHIGAMI, Junya. "Pabellón de Japón, XI Exposición Internacional de Arquitectura, Bienal de Venecia". El Croquis 184, Christian Kerez (2010-2015), Junya Ishigami (2005-2015). 2016, 184, pp. 180-181.

07. De manera no intencionada, el Pabellón de Venecia adquirió una imagen de estilo japonés debido a la similitud con la tradición ikebana y shakkei en la calculada disposición de las plantas y en el diseño de un jardín que se apropiaba de la preexistencia. La tradición ikebana data del siglo VII. Es un antiguo arte que emerge de un respeto hacia la naturaleza y está profundamente arraigado en la cultura japonesa, al igual que la caligrafía, la ceremonia del té y la poesía haiku. La técnica del shakkei, de origen chino, se introdujo en los jardines japoneses en el siglo XVII. Denominado en inglés como borrowed scenary, consiste en la apropiación de un escenario mediante el diseño de un jardín que incluye los objetos preexistentes.

08. HUNT, John Dixon. Greater Perfections The Practice of Garden Theory, Philadelphia, University of Pennsylvania Press, 2000, p. 273.

09. La estructura del Pabellón de Venecia se diseñó en colaboración con Jun Sato Structural Engineers, responsable del diseño de la estructura de otros proyectos de Ishigami como Balloon (2007) y House with Plants (2012).

10. Taro Igarashi, al hilo de las pinturas del interior del pabellón enfatiza su consideración como pieza de arte: "En el campo del arte uno puede encontrar elementos mínimos repetidos de forma obsesiva, como trabajos de Outsider Art, o artistas como Yayoi Deki, pero no en la representación de arquitectura". IGARASHI, Taro. "A Few Things I Know About Junya Ishigami". En Junya. Junya Ishigami: another scale of architecture, Kyoto, Seigensha Art Publishing, 2010, p. 282.
11. Se trata de exposiciones monográficas con temas que exploran nuevas arquitecturas: "Another scale of architecture" (Toyota Municipal Museum of Art, Toyota, 2010), "How small? How vast? How architecture grows" (Shiseido Gallery , Tokio, 2010) y "Junya Ishigami: petit? grand? l'espace infini de l'architecture", deSingel, Amberes, 2013 y Arc en Rêve, Burdeos, 2014.

12. La memoria del proyecto Sky reconoce la incapacidad tecnológica actual para llevar a cabo estos modelos. Además, hace referencia al carácter utópico de la propuesta: "Para ir más allá de las proporciones convencionales, con edificios infinitamente finos y altos, no consiste tanto en construir sobre el suelo como construir en el cielo. (...) En esto, un nuevo mundo, hasta ahora desconocido, está seguramente esperando". ISHIGAMI, Junya, Junya Ishigami: another scale of architecture, Kyoto, Seigensha Art Publishing, 2010, p. 135.

13. Ishigami incorpora un amplio y variado rango de referencias, como los primeros invernaderos de Salomon de Caus en Heildelberg (1619), ilustraciones de los años setenta de la artista americana Nancy Graves, mapas antiguos de la compacta ciudad de Srinigar junto al Himalaya, análisis de aviación a partir de la morfología de las aves o estudios de botánica sobre el perfil de bosques tropicales. Varias de estas referencias se incluyen en: ISHIGAMI, Junya, Junya Ishigami: another scale of architecture, Kyoto, Seigensha Art Publishing, 2010; e ISHIGAMI, Junya, Junya Ishigami: Small images, Tokyo, INAX, 2008.

14. La exposición y catálogo Another scale of architecture presenta esa diversidad de escalas a través de dibujos que abarcan desde las capas de la atmósfera hasta la estructura de una gota de agua. El libro recoge esos esquemas a través de cinco capítulos: nubes, bosque, horizonte, cielo, Iluvia. ISHIGAMI, Junya. Junya Ishigami: another scale of architecture, Kyoto, Seigensha Art Publishing, 2010.

15. En la defensa por una arquitectura libre de funciones uniformes, Ishigami define el término "no-uniformidad" como flexibilidad ambigua, es decir, aquella que no puede considerarse en un marco de expectativas concretas. ISHIGAMI, Junya. "De la libertad en arquitectura", El Croquis 184, Christian 


\section{Referencias bibliográficas}

Kerez (2010-2015), Junya Ishigami (20052015). 2016, 184, p. 158.

16. Ishigami traduce el concepto de "incertidumbre" en arquitectura como la cualidad de un sistema donde el mecanismo de cambio también se encuentra en transición ISHIGAMI, Junya. "De la libertad en arquitectura", El Croquis 184, Christian Kerez (2010-2015), Junya Ishigami (2005-2015), 2016, 184, p. 158.

17. Julian Worrall, en el artículo "The Deep Field: Resolving a Japanese Constellation" incluido en el catálogo de la exposición del MoMA de 2015, hace referencia al concepto "publicness" y sus connotaciones de política radical. GADANHO, Pedro. $A$ Japanese Constellation, New York City, The Museum of Modern Art, 2015, p. 216.

18. Taro Igarashi define el pabellón de Ishigami como una nueva arquitectura en sí misma, lejos de ser una pieza que replica el pasado: "Tal y como ejemplificaron en su día el Palacio de Cristal en la Gran Exposición de Londres de 1851 o el Pabellón de Barcelona de Mies en la Exposición Internacional de 1929 en España, las exposiciones internacionales son lugares donde la arquitectura experimental es presentada públicamente, abriendo el camino a nuevas posibilidades". IGARASHI, Taro, "A Few Things I Know About Junya Ishigami”. En Junya Ishigami: another scale of architecture. Kyoto, Seigensha Art Publishing, 2010, p. 283.

19. En Junya Ishigami: another scale of architecture, Kyoto, Seigensha Art Publishing, 2010, p. 4.
-AA.VV. JA 79, Junya Ishigami. 2010.

-BOSE, Shumi. "Architecture as Air: Château La Coste", Domus, 2011, Consultado el 16 de Julio de 2018, https://www.domusweb.it/en/ architecture/2011/08/03/architecture-asair-chateau-la-coste.html

- GADANHO, Pedro, $A$ Japanese Constellation, New York City, The Museum of Modern Art, 2015.

- IGARASHI, Taro, "A Few Things I Know About Junya Ishigami”, en: Junya Ishigami: another scale of architecture. Kyoto, Seigensha Art Publishing, 2010.

-ISHIGAMI, J., Junya Ishigami: Plants \& architecture, Tokyo, Junya. Ishigami+Associates, 2008.

-ISHIGAMI, J., Junya Ishigami: Small images, Tokyo, INAX, 2008.

- ISHIGAMI, J., "Greenhouse designing with Yoshizaka”, en: JA 79, Junya Ishigami, 2010.

- ISHIGAMI, J., Junya Ishigami: another scale of architecture, Kyoto, Seigensha Art Publishing, 2010.

- ISHIGAMI, J., Junya Ishigami: my works. Etsam, 16 de noviembre de 2011, Vídeo. Madrid, Servicios Audiovisuales, Etsam, 2011.

- ISHIGAMI, J., Junya Ishigami: how small? how vast? how architecture grows, Ostfildern, Hatje Cantz, 2014

- ISHIGAMI, J., "De la libertad en arquitectura", en: El Croquis 184, Christian Kerez (2010-2015), Junya Ishigami (20052015), 2016.

- ISHIGAMI, J., "Pabellón de Japón, XI Exposición Internacional de Arquitectura, Bienal de Venecia", en: El Croquis 184, Christian Kerez (2010-2015), Junya Ishigami (2005-2015), 2016.

- ISHIGAMI, J., "KAIT Workshop", El Croquis 184, Christian Kerez (2010-2015), Junya Ishigami (2005-2015), 2016.

- HUNT, John Dixon, Greater Perfections The Practice of Garden Theory, Philadelphia, University of Pennsylvania Press, 2000.
- LEVENE, Richard; MÁRQUEZ, Fernando, El Croquis 184, Christian Kerez (2010-2015), Junya Ishigami (2005-2015), 2016.

- OTERO, Marina, "125 hectáreas de arte. Château La Coste, un paisaje de arquitecturas", en: Arquitectura Viva, n. 146, 2012.

- WORRAL, Julian, "The Deep Field: Resolving a Japanese Constellation”, en: $A$ Japanese Constellation, New York City, The Museum of Modern Art, 2015.
RA. Revista de Arquitectura Núm. 20 - 2018

P. 192-203 